\title{
Integrando reflexiones sobre teoría y práctica de un caso en estudio
}

\section{Integrating reflections on theory and practice of a case study}

\begin{abstract}
Dra. María Inés Vázquez
Doctora en Educación, Universidad Autónoma de Barcelona, España. Master en Investigación Educativa, Centre International d'Études Pédagogiques (CIEP) - International Development Research Centre (IDRC), Canadá. Maestría en Sociedad e Instituciones, Universidad Nacional de San Luis, Argentina. Psicóloga. Consultora en Gestión Educativa. Investigadora, Consejo de Educación Secundaria. Integrante, Comisión Directiva del Instituto Nacional de Evaluación Educativa (INEEd). Coordinadora Académica de Gestión Educativa, Instituto de Educación, Universidad ORT Uruguay. Investigadora Nivel I - Sistema Nacional de Investigadores (SNI).
\end{abstract}

Mag. Carlos Varela

Master en Gestión Educativa, Universidad ORT Uruguay. Maestro. Integrante del Consejo Consultivo, Programa ProRazona, Consejo Directivo Central (CODICEN). Especialista en el área de la Didáctica de la Matemática. Maestro Director, Departamento de Educación Inicial y Primaria, Instituto Crandon.

Fecha de recibido: 17/02/2014

Fecha de aceptación: 11/06/2014

\section{Resumen}

Este artículo se basa en un estudio realizado en 2012 en el marco de la Maestría en Gestión Educativa del Instituto de Educación de la Universidad ORT Uruguay. La investigación analiza el rol de un directivo del sector primaria de un centro de educación privada.

Este trabajo integra aportes de dos investigadores con el propósito de generar una suerte de "diálogo" entre aspectos conceptuales y metodológicos, y los brindados desde el relato de una investigación específica.

La metodología utilizada es el estudio de casos, que permite caracterizar el escenario en estudio integrando diversos aspectos de la complejidad organizacional.

A través de diferentes técnicas como la entrevista, la observación, el análisis documental, encuestas y grupos de discusión, se procuró dar respuesta a algunas interrogantes: ¿Director y líder pedagógico?; ¿La institución educativa cambia o simplemente se "maquilla"?; ¿Se evalúa o se mira en un espejo?

Entre los principales hallazgos de esta investigación podemos afirmar que este centro pudo gestionar la crisis que lo llevó a replantearse cómo venían funcionando, lo que posibilitó la puesta en marcha de estrategias que abrieron puertas a nuevos desafíos.

PALABRAS CLAVE: liderazgo pedagógico - mejora escolar - comunidad de aprendizaje

\section{Abstract}

This article is based on a study conducted in 2012 as part of the Master Degree in Educational Management, Education Institute, ORT University, Uruguay. The research analyzes the role of a headmaster in a primary school of a private education center.

This work integrates contributions from two researchers with the purpose of generating a sort of 'dialogue' between conceptual and methodological aspects, as well as others provided by a specific research.

The methodology used is case study, allowing the characterization of the scenario under study by integrating various aspects of the organizational complexity. 
Various techniques such as interviews, observation, document analysis, surveys, and focus groups were used to try to answer to some questions such as: Headmaster and pedagogical leader? Does the educational institution change or simply "applies makeup" on the situation? Do we actually evaluate or just look ourselves in a mirror?

Among the main findings of this research we can state that this center was able to manage the crisis that led to a reconsideration of how they were working, which enabled the implementation of strategies that opened doors to new challenges.

KEYWORDS: instructional leadership - school improvement - learning community

\section{Introducción}

La metodología de casos representa un abordaje de investigación cuya principal relevancia es la de facilitar la comprensión de situaciones o fenómenos que se producen en contextos específicos.

Esta modalidad permite analizar a nivel particular aspectos sobre los que ya existe información desde otras perspectivas globales o universales (Cora, 2000). Con el propósito de aportar al conocimiento científico, resulta especialmente relevante que la temática central del o de los casos en estudio se asocie con tópicos ya detectados como relevantes desde otros estudios macroestructurales, o que hayan sido problematizados desde la teoría.

El estudio de casos requiere del registro y descripción densa de los diferentes componentes que forman parte del escenario en estudio (región, centro, sector o unidad), que permitan caracterizar a ese espacio como unidad de análisis desde el cual contextualizar al fenómeno en cuestión.

Es importante destacar que en este tipo de estudios la definición del problema surge del análisis de la propia realidad, no siendo el investigador quien define a priori la temática a abordar.

Tal como lo afirman Wainerman y Sautu (2011), los procedimientos metodológicos seleccionados para el estudio guardan relación con las perspectivas teóricas desde las cuales se definen los marcos explicativos y los criterios para el análisis de los datos. Desde este enfoque y tal como queda en evidencia en este caso a partir del relato que realiza el investigador, es posible identificar algunos criterios generales para la definición y organización inicial de la tarea. A modo de punteo se destacan especialmente los siguientes:

- La definición de un abordaje metodológico específico,

- La explicitación de ciertos referentes teóricos que encuadren las perspectivas de abordaje y den sentido a los procesos,

- La definición de una serie de criterios metodológicos y dispositivos para el relevamiento y análisis de los datos,

- La triangulación metodológica como estrategia para contrastar procedimientos e interpretaciones.

A fin de exponer el presente trabajo se ha estructurado una suerte de diálogo con el investigador, a lo largo del cual iremos desarrollando las diferentes fases de la investigación.

\section{¿Cómo se acercaron estos aportes a la práctica?}

Las reflexiones que se presentan en este trabajo surgen a partir del proceso que se realizó en una institución educativa en la cual se desarrolló una investigación en el marco de un proceso de mejora. A partir de la realización de un estudio diagnóstico para detectar los posibles factores causales asociados a una cierta problemática en estudio, se trabajó en un proceso de ida y vuelta con el centro, enriquecido por la reflexión permanente del equipo docente que participó en la elaboración de un Plan de Mejora. 
Para conocer a la institución se procedió a realizar una primera "fase exploratoria" que permitió un primer acercamiento a la estructura organizativa y al estilo de las dinámicas organizacionales así como a la identificación de los focos de preocupación de actores involucrados con el centro. Ello fue posible a través de diversas entrevistas con personas consideradas clave en la institución. A partir de las entrevistas exploratorias iniciales realizadas a distintas autoridades de la institución fue posible identificar la demanda del centro asociada al interés por registrar los avances que realizan los niños en sus aprendizajes.

Durante la segunda fase que denominamos de "comprensión del problema", y luego de haber identificado la demanda del centro, se realizó una nueva recolección de datos a partir de encuestas, análisis de diversos documentos, registros de observación y la realización de un grupo de discusión con docentes del nivel. A sabiendas de que las escuelas son instituciones donde los cambios se dan en forma lenta, se procuró no generar entre los actores institucionales una sensación de inseguridad así como no despertar en ellos falsas expectativas. Para ello fue fundamental mantener un diálogo fluido y franco tanto con la dirección como con los docentes, el cual permitió obtener informaciones pertinentes y confiables que permitieron justificar las decisiones tomadas. Este proceso participativo de evaluación diagnóstica implicó el inicio de un proceso de aprendizaje y cambio para la organización.

Rodríguez Mansilla (1998) propone al diagnóstico organizacional como un co-diagnóstico, lo que implica que el investigador tiene que colaborar junto a los miembros de la organización para dilucidar el problema así como sus posibles alternativas de solución. Teniendo en cuenta esta idea siempre se trabajó en permanente comunicación con la Directora así como con el resto de los integrantes del equipo de gestión.

Una estrategia que resultó fundamental en esta experiencia fue tomarse tiempo para identificar aspectos de la cultura organizacional: escuchar a los actores para conocer el contexto en el cual desarrollan sus actividades; conocer su forma de percibir, pensar y actuar; comprender los valores que subyacen en su día a día. Al respecto, Manes (1999) plantea que al realizar una intervención institucional es importante no descuidar aspectos de la cultura singular que comparten los actores de la organización para no generar conflictos innecesarios que puedan atentar contra el equilibrio en el que funcionan. Los cambios en el sistema organizacional se producen en forma permanente adaptándose constantemente a partir de variaciones internas y del entorno.

Rodríguez Mansilla (1998) sostiene que las organizaciones son sistemas determinados estructuralmente. Esto implica que el cambio solamente se posibilita dentro de la propia estructura organizacional. El entorno en el cual se encuentra la organización incide en la misma, por eso se plantea la necesidad de pensar en el cambio contextualizado ya que dos entornos diferentes provocarán cambios también diferentes. Es necesario conocer la situación en la que se encuentra un centro para intentar cambios en su estructura. "Una organización no puede ser cambiada, es la organización la que cambia" (Rodríguez Mansilla, 1998, 40). Es a partir de los propios elementos constituyentes de la organización que ésta puede cambiar.

Dos problemas enfrenté como investigador cuando se dio comienzo a la fase diagnóstica: uno fue la profusión de datos que van surgiendo a medida que se avanza con el relevamiento (datos que deben ser organizados y ponderados, jerarquizando lo importante y separándolo de lo secundario). Otro problema fue el manejo de los tiempos. Vinculado al tema del tiempo, Onetto (2010) escribe "Aceptar la temporalidad es entender que llegamos a una escuela que ya tiene un recorrido, que tiene costumbres, reglas, acuerdos (...) Aceptar la temporalidad es también aceptar que las cosas son así pero podrían haber sido de otro modo" (Onetto, 2010, 93). 
Como investigador y asesor debí tener en cuenta que la innovación no puede ser encarada como una ruptura total con el pasado, el cual representa la identidad de la institución y de los actores institucionales. Fue necesario tener presente que los cambios que desarrollan las personas surgen, generalmente, a partir de una negociación entre pasado y presente.

En relación al tema del tiempo es interesante la propuesta que realiza Manes (1999) sosteniendo que si bien el cambio es gradual, el mismo debe ajustarse a las necesidades internas y de afuera. No realizar los cambios a tiempo puede significar la pérdida de oportunidades que tal vez no se repitan.

A partir de las ideas de Gairín (2006) fueron varios los aspectos que como asesor tomé en cuenta al realizar el diagnóstico organizacional: la comunicación, la participación, el conflicto, la dirección, el liderazgo, la cultura organizacional, el clima de la organización. Todos estos conceptos fueron atendidos en su interrelación.

En el siguiente cuadro se presentan los conceptos que según Rodríguez Mansilla es necesario tomar en cuenta para armar un modelo del proceso de cambio.

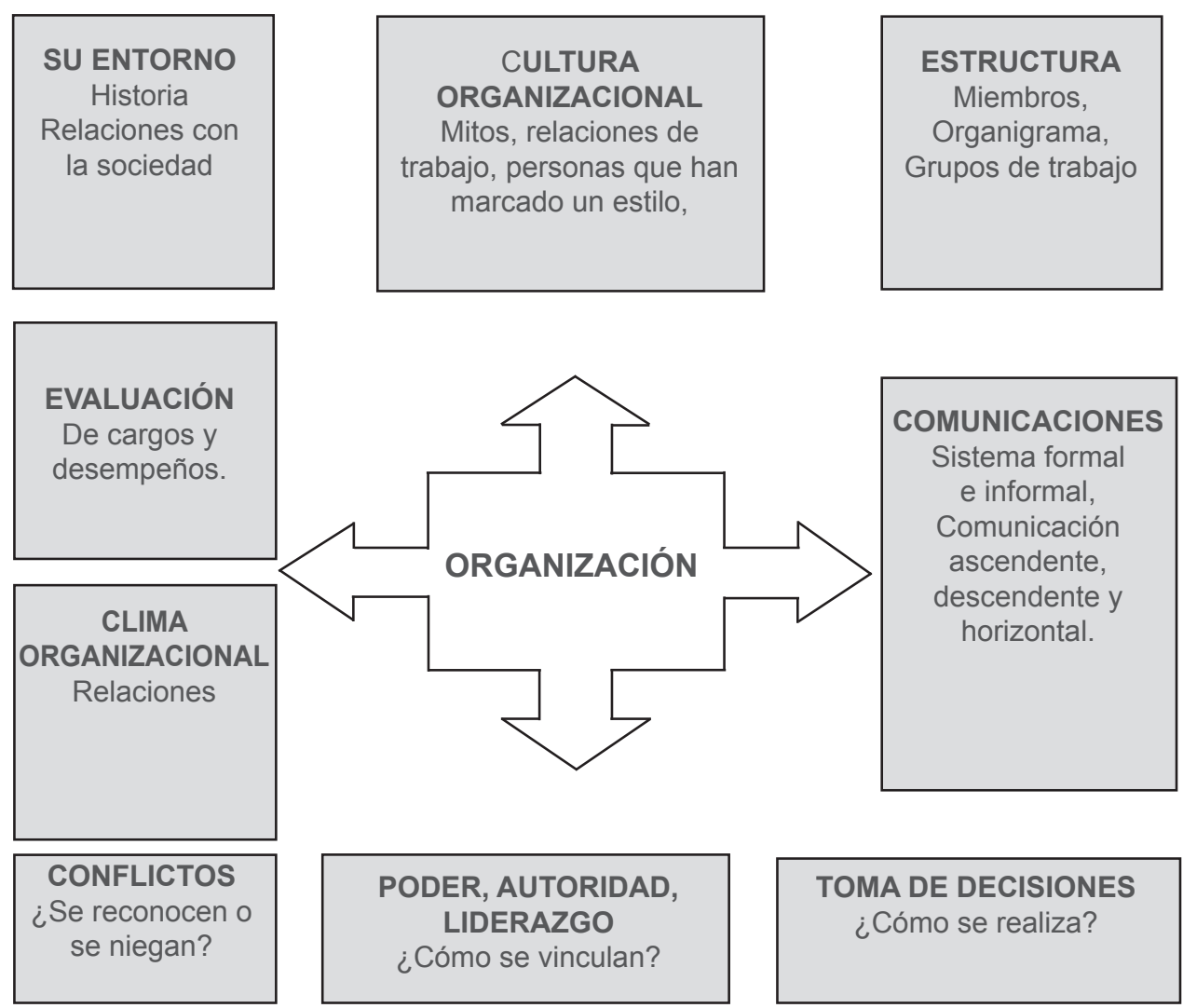

Realizar el diagnóstico de esta institución implicó una intervención en el sentido que tiene en latín el término interventio, que significa "venir entre, interponerse". La intervención implica un quiebre en la organización. Nicastro (2006) dice que "la intervención implica un recolocarse una y otra vez en espacios, presencias, miradas" (2006, 33). Para que esta intervención sea efectiva es necesario realizar un diagnóstico que permita conocer el estado concreto de esa organización. Si se toma en cuenta que el diagnóstico es una intervención, la organización que se propone llevarlo a cabo debe haber asumido la decisión de introducir cambios en la misma. 
Iniciar este proceso de diagnóstico implicó, al decir de Larrosa (2003), un acto de interrupción. Se entiende este acto como el llegar al descanso al subir una escalera. No significa que el proceso se detiene, que todo se suspende. Es una interrupción para mirar dónde y cómo se está para luego seguir avanzando. Nicastro $(2006,20)$ complementa esta idea al expresar que "En tanto intérpretes situados en ese volver a mirar la escuela, esperamos permitirnos mirar qué se mira, cómo se mira, para qué, desde qué lugar; sabiendo de antemano que se trata también de la implicación que nos envuelve y de la necesidad de hacernos cargo de nosotros mismos".

Es importante tener presente que al volver a mirar la escuela o hacer un nuevo reconocimiento de ella no pude limitarme a un simple ejercicio durante el cual nada fuera preguntado o cuestionado. Se plantearon dudas y preguntas, se movilizaron inquietudes; miramos con ojos de exploración y descubrimiento la cotidianeidad. Volver la mirada sobre la escuela no implica quedarse mirando el pasado sino que supone una manera diferente de mirar el presente. Implica también habilitar el no saber, el no tener todas las respuestas, el pensar en otras posibles.

Argyris (1999) plantea que para diagnosticar e intervenir en la organización el asesor tendría dos tareas fundamentales: por un lado ofrecer una explicación global que pueda dar cuenta de lo observado en la organización, una explicación que resulte coherente y verificable para los actores que participaron en el proceso de diagnóstico. Por otro lado tendría que poder presentar un plan de intervención (mejora), que pudiera romper con las rutinas defensivas de la organización y posibilitar el aprendizaje a todos los involucrados.

Rodríguez Mansilla (1998) también señala que la descripción que realice el asesor sobre la organización debe estar redactada de manera simple aunque se refiera a la complejidad de una organización. Fue conveniente señalar algunos aspectos del funcionamiento organizacional y rechazar otros, enfatizando la información que se consideró de mayor importancia para el contexto de esa escuela. Es fundamental que la organización pueda hacer suyo el diagnóstico elaborado para así poder adoptar decisiones que apunten a los cambios. "El estudio de la organización no debe, en ningún caso, perder de vista que es un estudio sistémico, vale decir, que está tratando de conocer un sistema complejo, inserto en un entorno societal. Cualquier análisis de una parte del sistema ha de considerar, necesariamente las intervinculaciones entre esta parte y el sistema, así como también las relaciones ambientales de la organización" (Rodríguez Mansilla, 1998, 37).

Fue crucial implicar a la institución en el proceso de diagnóstico organizacional para de esa manera proceder a la construcción de un saber compartido, un saber con la institución. Se procuró generar un proceso de construcción colectiva sobre una realidad que si bien era conocida para los actores institucionales, inicialmente, era desconocida para el investigador.

Identificada la demanda de la institución se pudo avanzar en la determinación del problema, el cual fue formulado como un marcado desequilibrio entre la propuesta académica y la formación en valores.

Al trabajar con los dispositivos matriz y modelo de análisis, se halló que eran dos las áreas que aparecían más comprometidas con la problemática: la dimensión organizacional y la dimensión pedagógico-didáctica (tomando la tipología de Frigerio y Poggi).

De una lectura realizada no al "pie de la letra" al decir de Bardin (1986) sino a partir de la búsqueda de interrelaciones entre los hallazgos, se pudieron establecer tres posibles factores causales asociados a este problema:

- Escasa visibilidad del liderazgo pedagógico

- Ausencia de criterios claros para la selección de las figuras clave (aquellas que desempeñan tareas vinculadas directamente a la Dirección de la escuela)

- Falta de estrategias para optimizar el uso del tiempo y los espacios del centro. 


\section{Marco teórico}

Los referentes teóricos seleccionados como sustento de la investigación son los que permiten definir las coordenadas conceptuales desde las cuales es abordado y analizado el fenómeno o situación en estudio. Ofician como "núcleo temático" a partir del cual se definen posibles "lentes" desde los cuales estudiar la realidad.

Esta estrategia de trabajo que procura desde el comienzo articular datos con aportes teóricos, sustituye otras modalidades basadas en la formulación de hipótesis que pretenden ser confirmadas durante la investigación a partir de los datos. Integra lo que Glaser y Strauss (1967) denominaron como "Teoría Fundamentada" (Grounded Theory), desde la cual entran en diálogo a través de la investigación saberes ya consolidados con otros que van emergiendo desde el propio estudio, permitiendo así el desarrollo de nuevos conocimientos. Estos investigadores refieren a una teorización sustantiva que identifican ligada al área empírica como principal espacio desde el cual enriquecer los saberes formales.

Estos referentes teóricos -así como los metodológicos- responden a ciertos marcos epistemológicos o conjunto de postulados interpretativos (Tesch, 1991) que definen los focos conceptuales y de interés desde los cuales el investigador aborda su estudio.

Los aportes teóricos representan una suerte de "hitos" en la definición, implementación y cierre de todo trabajo de investigación, fundamentalmente en tres momentos:

Al definir las coordenadas teóricas que ponen en evidencia la perspectiva seleccionada por el investigador para llevar adelante un estudio,

Al analizar los datos, triangulando las evidencias con referentes teóricos,

Al cerrar el trabajo, donde se explicita qué aportación realiza el estudio a la construcción de teoría.

En definitiva, es el carácter acumulativo de la investigación empírica el que permite salir de ciertas relaciones de inherencia que concentran y fundamentan la explicación de la realidad a partir de enunciados macroestructurales que más que a la comprensión tienden a la generalización de perspectivas y saberes.

El estudio de casos más que promover altos niveles de generalización procura una mayor comprensión del objeto de estudio en un nivel analítico.

\section{¿Cuáles fueron los conceptos teóricos seleccionados para este estudio?}

A partir de este momento empezaron a plantearse algunas coordenadas teóricas que permitieran focalizar en la comprensión y el análisis del problema. A través de la lectura y de la reflexión personal se pretendió dar respuesta a cuatro preguntas que se consideraron fundamentales para atender el problema de la institución:

1. ¿Director o líder educativo?

2. ¿Cambio educativo o maquillaje escolar?

3. Función de la escuela: provocar aprendizajes. Pero... ¿esta escuela aprende?

4. ¿Autoevaluarse o mirarse en un espejo?

La figura de un líder educativo es fundamental para lograr que los cambios puedan realizarse en las instituciones educativas sin ser ellos una mera fantasía como expresa Manes (1999).

Para que este cambio sea real, tendrá que incidir en la cultura organizacional. Es así que Fullan (2002) habla del concepto de reculturización de las escuelas. Esta reculturización será factible si el cambio se contextualiza a la realidad de cada organización y si existe en la misma un líder educativo que pueda impulsarlo y sostenerlo; proceso que tendrá que incidir directamente en los aprendizajes, tanto de los alumnos como de la propia organización.

Para adecuarse a los tiempos actuales, las escuelas tienen que transformarse en organizaciones inteligentes, en organizaciones que aprenden. Habrá que pensar en escuelas que sean capaces de buscar respuestas a los problemas que enfrentan sin considerar soluciones estereotipadas. Escuelas que trabajen como verdaderas comunidades de aprendizaje en las cuales los docentes no están aislados sino que se sienten parte del mundo. Escuelas que 
puedan reconocer sus errores y ser creativas en la búsqueda de soluciones a los mismos. Escuelas donde las rutinas se dejen de lado y se trabaje en la construcción de una visión de futuro compartida. Escuelas abiertas a la mejora y al cambio.

Álvarez Méndez (2005) señala que "todo parece vivir en la escuela bajo la atenta mirada del ojo evaluador". Desde la teoría se ha transitado desde un modelo de evaluación como control a un enfoque que valora la evaluación para la comprensión y la mejora; se plantean procesos de evaluación que estén vinculados con los procesos de enseñanza y aprendizaje; se plantea la necesidad de realizar procesos de autoevaluación tanto de los docentes y alumnos como a nivel institucional sin dejar de tener presente que la evaluación institucional y los procesos de cambio tienen que verse como complementarios. Es importante tener presente una idea que plantea Santos Guerra: "En la escuela se desarrolla, a mi juicio, un fenómeno curioso y sorprendente: es el lugar donde se evalúa con más frecuencia y se cambia con menos rapidez" (1995, 71).

\section{El trabajo de campo}

El trabajo de campo propiamente dicho integra tanto en su planificación como en su puesta en marcha, una serie de componentes que deben ser tenidos en cuenta, entre los cuales destacamos cuatro:

Componente "estratégico". Investigaciones de tipo etnográfico como las que aquí se relatan, que requieren de altos niveles de involucramiento de los integrantes del centro que oficiará como "caso" de estudio (Cora, 2000). Son procesos que, partiendo del caso que nos ocupa, tienen una duración promedio de ocho meses, en los cuales la interacción del investigador con distintos actores educativos (ya sea durante la etapa diagnóstica o de elaboración del plan de mejora) resulta sustancial. En tal sentido y antes de formalizar el inicio del estudio, el investigador ha de cerciorarse de que al centro efectivamente le interesa llevar adelante este proceso desde una posición activa y participativa.

Componente "confianza". Abrir las puertas del centro habilitando el ingreso a personas que tienen como principal objetivo registrar y analizar lo que allí sucede no representa una situación común. En este sentido es fundamental explicar de antemano cómo se piensa realizar la recolección de datos, qué criterios éticos serán tenidos en cuenta a la hora de procesar y manejar la información obtenida, aclarando que se trabaja bajo el principio de confidencialidad.

Componente "implicancia". El proceso de trabajo con la institución implica una primera fase diagnóstica y una segunda fase de plan de mejora. Para llevar adelante cada fase dentro de los plazos previstos resulta fundamental contar con la implicancia de quienes participarán en el proceso (Sosa Escudero, 2011), tanto para poner a disposición los datos requeridos como para otorgarle sentido al proceso que la propia institución valida en la definición del plan de mejora.

Componente "retroalimentación". Como estrategia que asegure mantener y consolidar los tres componentes anteriores, se entendió necesario asegurar mecanismos de retroalimentación que permitieran mantener en alto el interés y la motivación de los implicados en el estudio (Vázquez, 2011). Es por ello que tanto al finalizar la fase diagnóstica como al terminar la elaboración del plan de mejora el investigador hace entrega al centro en estudio de un documento breve con los principales aspectos de la etapa cumplida. En muchos casos, como en el integrado en este relato, ante la entrega de los documentos el centro solicita al investigador que lo presente formalmente a otros integrantes de la comunidad educativa de esa institución. 


\section{¿Cuáles fueron las características del trabajo de campo?}

Con los aportes del marco teórico y luego del análisis de los elementos recogidos en la primera etapa exploratoria se organiza una segunda recolección de datos identificando nuevos actores clave, para lo cual fue necesario diseñar otros instrumentos para la recolección de información complementaria.

Los nuevos informantes elegidos fueron los maestros de clase y los alumnos de $6^{\circ}$ año. Los maestros fueron propuestos por la Dirección de la escuela y representan los diferentes grados escolares. Para el trabajo con ellos se preparó un Focus Group presentándole diferentes planteos que fueron extraídos de las entrevistas inicialmente realizadas y sobre los cuales se pidió su opinión. Los alumnos de $6^{\circ}$ año fueron elegidos pues son los que poseen un mayor conocimiento de la realidad de la escuela y quienes podían aportar una reflexión más profunda sobre la misma. Con tal propósito se diseñó una encuesta semiestructurada en la que se abordaron los diferentes temas que surgieron en las entrevistas exploratorias.

En esta etapa se analizó también la página Web de la institución con el propósito de identificar los aspectos que la institución jerarquiza y aquellos otros sobre los cuales no aparece ninguna información.

Luego de disponer de todo el material y de proceder a la lectura y relectura del mismo se comenzó el análisis de la información, con el fin de encontrar aquellos elementos que permitieran profundizar la comprensión de la realidad de la institución.

Según Tójar $(2006,285)$ "El análisis cualitativo implica ordenar y organizar la información disponible, además de orientar su búsqueda, elaborar patrones, categorías y unidades de análisis con los que reorganizar las primeras unidades seleccionadas. Pero analizar cualitativamente supone también interpretar, asignar significados, describir y comprender los patrones encontrados y buscar conexiones entre éstas y otras categorías.(...) analizar cualitativamente incluye valorar, elaborar y razonar juicios sobre criterios que se han hecho explícitos, usando claves internas de interpretación".

Se realizó luego una triangulación de las evidencias a partir de las distintas fuentes (Maestra Directora, coordinadores, maestras, niños, documentos). También se triangularon los datos obtenidos desde las diferentes técnicas (observación, entrevistas, Focus Group, encuestas). La triangulación cumple la función de protegernos de una visión única, permitiendo confrontar y someter a control recíproco distintas fuentes de información. De esta manera se fortalece la validación de los hallazgos así como el grado de confianza logrado. A modo de ejemplo se integran aquí algunos aportes representativos del conjunto: 


\begin{tabular}{|c|c|c|}
\hline Preguntas & \multicolumn{2}{|c|}{ Algunas evidencias } \\
\hline ¿Director o líder educativo? & $\begin{array}{l}\text { "...Veíamos como una ausencia, } \\
\text { nos sentíamos como un poco } \\
\text { que no había de parte de la } \\
\text { Dirección... un seguimiento } \\
\text { de los avances o un apoyo en } \\
\text { las coordinaciones, en el aula } \\
\text { también, ¿no?" (Maestra) }\end{array}$ & $\begin{array}{l}\text { "Uno queda en las cosas medio } \\
\text { macras, medio generales y cómo } \\
\text { también apoyar en lo pequeño, } \\
\text { en lo chico, que por ahí durante } \\
\text { el año, pero a veces también } \\
\text { las instancias no terminan como } \\
\text { siempre de encajar, no tenés } \\
\text { tiempo en la dinámica" (M. } \\
\text { Directora) }\end{array}$ \\
\hline $\begin{array}{c}\text { ¿Autoevaluarse o mirarse en } \\
\text { un espejo? }\end{array}$ & $\begin{array}{l}\text { "Evaluar el nivel académico de } \\
\text { cada ciclo" (Informe de Asesoría } \\
\text { Externa). }\end{array}$ & $\begin{array}{l}\text { "No, él es más para este colegio } \\
\text { porque... el hermano que puede } \\
\text { ser exigido más sigue en el otro } \\
\text { colegio, como que en el otro } \\
\text { colegio exigen y acá no... es una } \\
\text { imagen" (Maestra) }\end{array}$ \\
\hline $\begin{array}{l}\text { ¿Cambio educativo o } \\
\text { maquillaje escolar? }\end{array}$ & $\begin{array}{l}\text { "También está el tema del } \\
\text { acuerdo en teoría y después lo } \\
\text { que tenés que aplicar en la clase. } \\
\text { Capaz que en teoría hay más } \\
\text { acuerdo de lo que en realidad } \\
\text { después sale cuando vas a la } \\
\text { cancha..." (Maestra) }\end{array}$ & $\begin{array}{l}\text { "Bueno, el viejo y querido } \\
\text { problema de los acuerdos, es } \\
\text { lo que más cuesta al docente. } \\
\text { Acuerdos ya sea pedagógicos } \\
\text { o acuerdos de funcionamiento". } \\
\text { (Maestro responsable del turno } \\
\text { matutino) }\end{array}$ \\
\hline ¿Esta escuela aprende? & $\begin{array}{l}\text { "Tenemos la necesidad de lo } \\
\text { académico empezar a mostrarlo } \\
\text { más y a mejorar, en ese sentido. } \\
\text { Logros académicos más } \\
\text { tangibles" (M. Directora) }\end{array}$ & $\begin{array}{l}\text { "Profundizar y mejorar los logros } \\
\text { y niveles alcanzados" (Informe de } \\
\text { Asesoría Externa) }\end{array}$ \\
\hline
\end{tabular}

De las evidencias analizadas, se pudo constatar que el liderazgo aparece como un punto débil en esta institución. Desde los datos, la Dirección aparece sin fuerza suficiente para contrarrestar otras fuerzas inerciales del centro que tienden a dejar todo como está. Coincidimos con Gairín (1996) en señalar que hay aspectos que hacen pensar más en un gestor que en un líder educativo. Este es un factor que dificulta los cambios y más aún el sostenerlos en el tiempo. Si bien los docentes manifiestan conformidad con la Dirección, los cambios no se producen. Esta incapacidad de revertir situaciones se refleja también en los aprendizajes tanto a nivel de los alumnos como a nivel de la propia institución. Parecería que esta escuela se limitara a mirarse en el espejo sin capacidad de identificar otras alternativas para hacer escuela.

\section{Del diagnóstico al plan de mejora: una tarea colectiva}

Esta metodología de trabajo permite al centro pasar de un nivel que podría denominarse "anecdótico" en el que se comenta sobre un problema recurrente que no se logra superar, a otro "operativo" en el cual -con la colaboración del investigador- se logra abordar el problema, identificar posibles factores causales que lo generan y definir un plan de trabajo para superarlo. 
El proceso de investigación e intervención realizado puede ser caracterizado como:

Investigación cualitativa, al destacar como principal propósito el de comprender una cierta situación humana en el marco de escenarios específicos, utilizando técnicas que permiten captar aspectos no siempre evidentes a simple vista.

Investigación aplicada, al procurar comprender la realidad en estudio para luego intervenir en ella (relación entre factores causales y propuesta de mejora).

Investigación explicativa, dado que busca identificar algunos aspectos que están asociados con la problemática que aparece como tema problemático para la institución en estudio.

Investigación ideográfica, al rescatar lo particular y único del caso en estudio buscando relacionar el tema en estudio con aspectos del contexto y con las representaciones que los actores implicados manifiestan tener sobre la problemática.

Tomando en cuenta los criterios que le dan sustento a esta metodología, se destacan los de:

- Fiabilidad de la propuesta. En ciencias sociales este criterio se logra disminuyendo en la medida de lo posible los sesgos personales del investigador. Para ello fueron elaborados tanto para el estudio diagnóstico como para el plan de mejora protocolos que contemplan las distintas fases de avance, las principales actividades previstas en cada fase así como los logros que se prevé alcanzar en cada caso. Asimismo, en la etapa de análisis, se dispone de una serie de dispositivos para el mismo (analizadores) cuya utilidad está asociada a distintos niveles de complejidad en el tratamiento de los datos.

- Validez (conceptual). Se logra al utilizar diversas fuentes de datos que permitan establecer "cadenas de prueba" (Yin, 2004). En el estudio de casos se utiliza en la fase exploratoria en la cual se contrastan visiones y también durante la segunda recolección de datos, en la cual se intenta triangular representaciones sobre un mismo tema en estudio. También se procura que el marco teórico que sustenta el estudio integre aportes desde diferentes perspectivas, algunas complementarias y otras hasta opuestas, articulando así diversos enfoques sobre el tema en cuestión.

- Validez (interna y externa). Refiere al uso de distintas técnicas que permitan la construcción de argumentos durante el proceso de análisis (Latorre y otros, 2005).

\section{¿Cómo se organizó el trabajo con la institución?}

Plantear la elaboración de un Plan de Mejora implicó pensar qué significa mejorar. En el Diccionario de la Real Academia Española, entre otras se encuentran estas dos acepciones:

- Adelantar, acrecentar algo, haciéndolo pasar a un estado mejor.

- Poner mejor, hacer recobrar la salud perdida.

En ambos significados aparece el término mejor, pasar a un "estado mejor", "poner mejor". Nuevamente el Diccionario proporciona el significado de este término: superior a otra cosa y que la supera en una cualidad natural o moral.

Se deduce entonces que elaborar un Plan de Mejora Organizacional implica diseñar un conjunto de estrategias de trabajo que permita que la organización estudiada mejore, crezca, avance en relación a cómo está en el momento del diagnóstico. Es decir que se procura que la organización pase de una situación real en la que se encuentra a una situación deseada, mejor. 
A lo largo del proceso realizado en la institución presentamos al equipo impulsor otras imágenes, compartimos los hallazgos que surgieron de las entrevistas, encuestas y del grupo de análisis de manera de brindar lo que el espejo no muestra. Todo ello con el fin de pensar soluciones a las debilidades reconocidas por el equipo, soluciones que fueran factibles de llevar a la práctica tales como focalizar el trabajo en los contenidos curriculares informando a las familias de este trabajo e incentivar los procesos de evaluación tanto de alumnos como de docentes.

En la elaboración del Plan de Mejora se trabajó con representantes de la institución para tomar en cuenta las lógicas de trabajo del centro, revisando alternativas que permitieran procesos de mejora exitosos. Este grupo impulsor tuvo un rol preponderante en esta fase, al conocer en profundidad a la institución y estar comprometido con ella y su mejora. Esta estrategia de trabajo permitió a su vez atender y trabajar posibles resistencias al cambio a las que refiere Gather (2004) "las investigaciones sobre el cambio en la educación han considerado la resistencia del colectivo docente como uno de los obstáculos principales” $(2004,14)$.

A partir de algunas ideas de Gather $(2004,11)$ se elabora un cuadro con las características de la institución que modifican la probabilidad del cambio.

\begin{tabular}{|l|l|l|}
\hline $\begin{array}{l}\text { Aspectos de la cultura y del } \\
\text { funcionamiento de la institución }\end{array}$ & $\begin{array}{l}\text { Características desfavorables } \\
\text { al cambio }\end{array}$ & $\begin{array}{l}\text { Características favorables al } \\
\text { cambio }\end{array}$ \\
\hline Organización del trabajo & $\begin{array}{l}\text { Organización rígida. Actitudes } \\
\text { individualistas. }\end{array}$ & $\begin{array}{l}\text { Organización flexible y } \\
\text { negociable. }\end{array}$ \\
\hline Relaciones profesionales & $\begin{array}{l}\text { Individualismo. Pocas } \\
\text { discusiones sobre temas } \\
\text { profesionales. }\end{array}$ & $\begin{array}{l}\text { Compañerismo. Intercambios } \\
\text { profesionales. }\end{array}$ \\
\hline Cultura e identidad colectivas & $\begin{array}{l}\text { La actividad profesional se } \\
\text { considera como una rutina. }\end{array}$ & $\begin{array}{l}\text { Predomina la reflexión sobre la } \\
\text { práctica. }\end{array}$ \\
\hline $\begin{array}{l}\text { Capacidad de proyección en el } \\
\text { futuro }\end{array}$ & $\begin{array}{l}\text { No todos los docentes adhieren } \\
\text { a un proyecto. }\end{array}$ & $\begin{array}{l}\text { La mayoría de los docentes } \\
\text { adhieren al proyecto. }\end{array}$ \\
\hline $\begin{array}{l}\text { Liderazgo y formas de ejercer } \\
\text { el poder }\end{array}$ & Autoridad burocrática. & Liderazgo cooperativo. \\
\hline $\begin{array}{l}\text { La institución como } \\
\text { organización que aprende }\end{array}$ & $\begin{array}{l}\text { La institución es un lugar de } \\
\text { trabajo. No importa el futuro. }\end{array}$ & $\begin{array}{l}\text { Modelo profesional. Desarrollo } \\
\text { de la calidad. Enfrentan los } \\
\text { problemas. }\end{array}$ \\
\hline
\end{tabular}

La elaboración del Plan de Mejora implicó la definición de los aspectos susceptibles de mejora, los objetivos generales y específicos, las líneas de acción que serían implementadas así como los logros proyectados. También se trabajó en el diseño de mecanismos para el seguimiento y la sustentabilidad del proceso.

Con el equipo impulsor del Plan de Mejora se procuró:

- identificar aspectos tanto estructurales como funcionales factibles de ser ajustados

- realizar seguimiento de estos procesos tomando en cuenta las metas previstas

- trabajar en forma colaborativa, coordinando esfuerzos en torno a las distintas líneas de trabajo proyectadas.

Como plantea Gairín, “la organización no sólo puede considerarse como un marco o agente educativo, también puede pensarse como el resultado de un proceso de mejora o como un objeto sobre el que se interviene. Hablamos de la organización que aprende cuando referenciamos un tipo de organización capaz de aprender de sus errores y de configurarse de manera distinta" (1999, 381).

Desde la institución de referencia se esperaban respuestas a los problemas cotidianos, a los hechos del presente. Intentamos siempre definir esas respuestas en forma conjunta, 
atendiendo a este presente pero sabiendo que la educación es una construcción de futuro. En esa tensión entre presente y futuro fuimos construyendo el Plan de Mejora con los pies en la realidad concreta de la escuela pero con nuestras mentes mirando hacia el porvenir. La elaboración de este plan pretendió pensar algunas nuevas respuestas frente a la necesidad de construir una nueva escuela que fuera capaz de modificarse pero sin descuidar su misión de transmitir el conocimiento. Procuramos fortalecer la figura de la Directora aportando algunas herramientas que la ayudaran a gestionar la escuela de una manera diferente.

\section{Perspectiva de análisis}

La perspectiva situada desde la cual opera el investigador le permite identificar, analizar y asignar sentido a una problemática concreta planteada desde el centro (caso), e identificar posibles alternativas para su mejora. Este encadenamiento de acciones permite promover pensamiento crítico en el colectivo institucional.

Para autores como Boisvert (2004), el pensamiento crítico se asocia con la predisposición para atender de manera reflexiva los problemas que surgen en la vida cotidiana. Este concepto bien podría ser trasladado a la disposición que las instituciones y sus equipos deberían tener para poder abordar de forma diferente problemáticas con las que conviven, en algunos casos, de forma casi crónica.

Según este autor, para poder generar reflexión crítica se requiere de la presencia de al menos dos características:

(i) una actitud apropiada (no estar a la defensiva, amplitud de criterios, honestidad profesional) y

(ii) capacidad de razonamiento y criterios para la investigación lógica.

Desarrollar pensamiento crítico implica transitar por un proceso que, además de ser colectivo en el caso de las instituciones, requiere de una íntima relación entre reflexión y prácticas. No todo podrá ser abordado al mismo tiempo ni resuelto de forma instantánea, pero sí es posible marcar un rumbo, identificar prioridades y negociar estrategias para su puesta en marcha.

Siguiendo a Brookfield (1987) las fases que organizan y consolidan la generación de pensamiento crítico son cinco:

- Identificación de una o más situaciones que generan incomodidad o preocupación.

- Indagación de la situación global (diagnóstico)

- Búsqueda de explicaciones y posibles soluciones

- Integración de diferentes perspectivas (alternativas de acción)

- Resolución de la/s situación/es

Desarrollar pensamiento crítico a nivel colectivo permite una mejor adaptación de las instituciones a las variaciones internas y a otras que marca el entorno.

\section{¿A qué conclusiones llegó el investigador?}

El desarrollo de la presente investigación nos acercó a la realidad de una institución educativa que abrió sus puertas a este estudio en la búsqueda de un camino que le permitiera mejorar aspectos sentidos como debilidades. En este proceso, que lejos de ser lineal tuvo sus idas y vueltas, fueron surgiendo interrogantes que nos llevaron a reflexionar y a acercarnos a la realidad desde distintas perspectivas. Ello nos obligó a identificar puntos de encuentro entre las distintas percepciones de la realidad pero siempre, al decir de Schvarstein (2004) con la esperanza de tener éxito ante el desafío enfrentado y que a diario otorga sentido a nuestra vida.

En este trabajo se buscaron respuestas a algunas preguntas que nos planteamos. En relación al liderazgo en la institución educativa partimos del entendido de que un buen líder educativo debe lograr ascendencia sobre su personal sin que ello afecte las relaciones profesionales y debe poseer la fortaleza de tomar decisiones a nivel individual aun sabiendo los costos que ello 
puede implicar. Si tomamos en cuenta las opiniones de los docentes a la luz de las ideas de Gairín (1999), podemos decir que un líder que evita las soluciones que pueden ser conflictivas hace más al rol de gestor que al rol de líder educativo. El director que es líder educativo debe gobernar los procesos de cambio y direccionar las prácticas del centro hacia la mejora que se aspira alcanzar.

Pensamos en un líder pedagógico centrado en los aprendizajes de los alumnos, de los docentes y de la escuela misma. Sabemos que no alcanza con encontrar los errores sino que hay que emprender las acciones que permitan subsanarlos; identificar nuevas respuestas para los problemas de siempre y, al decir de Blejmar (2005), fundamentalmente lograr que las cosas sucedan.

Liderazgo pedagógico implica corrección de los errores, nuevas respuestas, escuelas organizadas de forma flexible, escuelas que procuran el cambio a partir de las propias necesidades, con prioridades claras, focalizadas en lo académico, con sistemas de evaluación permanente; escuelas que generan un clima de trabajo estimulante y positivo. Sostenemos que éstas son las características de una escuela inteligente, una escuela que aprende, una escuela que poco a poco es generadora de conocimiento, escuelas en las que se construye futuro.

Si evidenciamos que la Dirección de la escuela no ejerce liderazgo educativo, es difícil que se puedan provocar cambios y más aún sostenerlos en el tiempo. Es necesario que las acciones se transformen en actos y para ello es necesario gestionar la escuela en el sentido de realizar intervenciones potentes ya que la ausencia de intervención también produce efectos. Si los cambios se establecen en el discurso, como lo plantean algunas docentes de la institución, pero no aparecen reflejados en las prácticas pedagógicas, hablamos de cambios "cosméticos" ya que quedan en la superficie y no llegan a modificar la cultura organizacional.

En una escuela en la que el liderazgo educativo es una debilidad y en la que no se pueden sostener los procesos de cambio introducidos, sin duda el camino que tendrá que recorrer para transformarse en una escuela que aprende será más largo y posiblemente lleve más esfuerzo y más tiempo. La escuela en estudio se encuentra abierta a los diagnósticos, pero para transformarse en una organización que aprende necesita ajustar los mecanismos de intervención y de evaluación permanente. Desarrollar estos mecanismos habilitará la formación de comunidades de aprendizaje que posibiliten los procesos de cambio.

Si nos limitamos a mirarnos en un espejo, siempre veremos nuestra misma imagen, pues el espejo nos permite ver lo que somos y no lo que queremos ser. El espejo no nos devuelve otras imágenes posibles sino que esas imágenes deben ser construidas por nosotros a partir de procesos de reflexión y de discusión entre todos los actores que participan de la vida de la escuela. Por eso es importante avanzar en el proceso de evaluación y autoevaluación tanto a nivel docente como a nivel institucional.

Es difícil gestionar las escuelas en una época de incertidumbres como la que vivimos, pero sostenemos que es posible. En relación a este tema, Aguerrondo (2007) escribe: "quien gestiona (planifica, toma decisiones) debe tener la capacidad de pasar de una conceptualización binaria del futuro (una determinada característica del futuro es o no es) a una de múltiples determinaciones. O sea, como punto de partida, es necesario poder distinguir diferentes incertidumbres" $(2007,15)$.

Algunas ideas pueden ayudarnos en este complejo y a veces solitario proceso que realizan los directores de las escuelas. Primeramente es necesario asumir que somos nosotros mismos los que construimos nuestro destino. Si buscamos otros responsables, si justificamos lo que sucede atribuyendo a otros la toma de decisiones, no habrá avances, no se realizarán los 
cambios necesarios. En segundo lugar, es importante generar mayores espacios de reflexión tanto personal como colectiva, disponer de tiempos para pensar en lo que sucede, en los porqués. En una época en que todo se hace en forma rápida, los directores de escuela tenemos que hacernos un espacio en nuestras agendas para la reflexión. La integración de equipos de trabajo es un elemento que favorece estos procesos reflexivos. En tercer lugar sostenemos que no podemos mantener imágenes idílicas del pasado sino que es imprescindible asumir el tiempo en el cual vivimos, con sus obstáculos pero también con sus múltiples posibilidades que hay que saber aprovechar. No olvidar el pasado ya que puede ser fuente inspiradora en el proceso de gestión, tener en cuenta la situación actual, el presente que vivimos para que podamos imaginar el futuro al cual queremos llegar.

Desde mi rol de Maestro Director de una escuela primaria, cargo que ejerzo desde hace 17 años, puedo afirmar que el liderazgo pedagógico del maestro director es un elemento clave, fundamental, para lograr los cambios en la escuela. Un director con la preparación técnica y pedagógica que lo habilite a generar en la comunidad educativa el respeto y la confianza necesarios para que su voz sea escuchada. Un director comprometido con el aprendizaje de alumnos y docentes, así como en su propio aprendizaje. Un director que sea capaz de escuchar, de oír, de acompañar, de dialogar, de trabajar en equipo, de amar.

Una de las riquezas mayores que tuvo este trabajo de investigación fue el diálogo, diálogo que se dio en forma simultánea entre el investigador y el equipo impulsor del Plan de Mejora y aquel que se fue generando también, al mismo tiempo, entre el investigador y el tutor que siempre tuvo su palabra certera, de guía, de apoyo al trabajo que se estaba realizando.

\section{Reflexión de cierre}

Al decir de Duch (2004), lo que caracteriza a la construcción social de la realidad (de la que los centros educativos forman parte) es la imperiosa necesidad de acercar y poner en común las distintas visiones que conviven en un mismo ámbito de acción (la de docentes, estudiantes, directivos, personal administrativo y de servicio, etc.).

Las estrategias metodológicas implementadas por el investigador han permitido tomar registros, analizar y compartir hallazgos con los actores institucionales implicados. Poner en evidencia las diferentes perspectivas, promover acuerdos, identificar prioridades, permite salir del plano anecdótico y pasar a otro operativo que habilite la puesta en marcha de acciones colectivas que aseguren mejoras. 


\section{Bibliografía}

Aguerrondo, I. (1998). Cómo será la escuela del Siglo XXI. En Filmus, D. Para qué sirve la escuela. Buenos Aires. Editorial Norma.

Aguerrondo, I. (2007). Racionalidades subyacentes en los modelos de planificación educativa. Texto elaborado a partir de la discusión en la mesa redonda sobre "30 años de planeamiento educativo", realizada durante el X Curso Regional sobre Formulación y Administración de Políticas Educativas, promovido por IIPE/UNESCO-Buenos Aires, setiembre 2007.

Álvarez Méndez, J. M. (2005). (2ª edición). Evaluar para conocer, examinar para excluir. Madrid. Ediciones Morata.

Argyris, C. (1999). Conocimiento para la acción. Una guía para superar los obstáculos del cambio en la organización. Barcelona. Ediciones Granica.

Blejmar, B. (2005). Gestionar es hacer que las cosas sucedan. Buenos Aires. Ediciones Novedades Educativas.

Boisvert, J. (2004). La formación del Pensamiento Crítico. Teoría y Práctica. México. Fondo de Cultura Económica.

Brookfield, S. (1987). Developing Critical Thinkers: Challenging Adults to Explore Alternative Ways of Thinking and Acting. San Francisco. J-B Publishers.

Cora, E. (2000). La recuperación del análisis institucional como perspectiva teórico-metodológica. En: Besse y otros. Topografía de la investigación. Buenos Aires. Eudeba.

Cora, E. y otros (2000). Topografía de la investigación. Métodos, espacios y prácticas profesionales. Buenos Aires. Eudeba.

Duch, L. (2004) Estaciones del laberinto. Ensayos de antropología. Barcelona: Herder

Frigerio, G.; Poggi, M. (1996) (6ª edición). Las instituciones educativas. Cara y Ceca. Elementos para su gestión. Buenos Aires. Troquel Educación.

Fullan, M. (2002). Los nuevos significados del cambio en la educación. Barcelona. Ediciones Octaedro.

Gairín Sallán, J. (1999) (2ª edición). La organización escolar: contexto y texto de actuación. Madrid. Editorial La Muralla.

Gairín Sallán, J. (2006). Procesos de cambio en los centros educativos a partir de evaluaciones externas. Madrid. Ministerio de Educación y Ciencia. Dirección General de Educación, Formación Profesional e Innovación Educativa.

Gather Thurler, M. (2004). Innovar en el seno de la institución escolar. Barcelona. Graó.

Glaser, B.; Strauss, A. (1967). The Discovery of Grounded Theory: Strategies for Qualitative Research. Chicago. Aldine Press.

Larrosa, J. (2003). Entre las lenguas. Barcelona. Laertes.

Latorre y otros. (2005). Bases metodológicas de la investigación educativa. Barcelona. Ed. Experiencias.

Manes, J. M. (1999). Gestión Estratégica para Instituciones Educativas. Buenos Aires. Ediciones Granica.

Nicastro, S. (2006). Revisitar la mirada sobre la escuela. Exploraciones acerca de lo ya sabido. Rosario. Homo Sapiens. 
Onetto, F. (2010). El tiempo pedagógico y el tiempo político. En Autonomía. El tiempo y el espacio pedagógicos para la escuela del S. XXI. Documento II. Montevideo. Administración Nacional de Educación Pública. Consejo de Educación Inicial y Primaria.

Rodríguez Mansilla, D. (1998) (3ª edición). Diagnóstico organizacional. Santiago de Chile. Pontificia Universidad Católica de Chile.

Santos Guerra, M. A. (1995) (2ª edición). La evaluación: un proceso de diálogo, comprensión y mejora. Málaga. Ediciones Aljibe.

Schvarstein, L. (2004). La inteligencia social de las organizaciones. Desarrollando las competencias necesarias para el ejercicio efectivo de la responsabilidad social. Buenos Aires. Paidós.

Sosa Escudero, W. (2011). Cuestiones metodológicas en una investigación sobre pobreza multidimensional en América Latina. En: Wainerman, C. y Sautu, R. (2011). La trastienda de la investigación. Buenos Aires. Manantiales.

Tesch, R. (1991). Software for Qualitative Researchers: Analysis Needs and Program Capabilities. London.

Tójar Hurtado, J. C. (2006). Investigación cualitativa. Comprender y actuar. Madrid. La Muralla. Vázquez, M. I. (2011). Procesos de cambio y autoevaluación en centros educativos. ¿Dos caras de una misma moneda? Barcelona. Da Vinci.

Wainerman, C. y Sautu, R. (2011). La trastienda de la investigación. Buenos Aires. Manantiales. Yin, R. (2004). Case Study Methods: Cosmos Corporation. Buenos Aires. Manantiales. 